\title{
Monogenic Lupus with IgA Nephropathy Caused by Spondyloenchondrodysplasia with Immune Dysregulation
}

\author{
Haseena Sait ${ }^{1} \cdot$ Harikrishnan Gangadharan ${ }^{2} \cdot$ Aviral Gupta $^{3} \cdot$ Amita Aggarwal $^{2}$ - Manoj Jain ${ }^{3} \cdot$ Shubha R. Phadke $^{1}$
}

Received: 28 August 2020 / Accepted: 18 December 2020 / Published online: 13 March 2021

(C) Dr. K C Chaudhuri Foundation 2020

\begin{abstract}
Monogenic disorders causing systemic lupus erythematosus represent a small subset of cases. Type-1 interferonopathies, like spondyloenchondrodysplasia with immune dysregulation constitute an important functional category of monogenic lupus. Apart from autoimmune disorders, neurological and skeletal abnormalities are additional manifestations observed in this disorder. A young female presented with seizures due to acute hemorrhagic stroke secondary to malignant hypertension. On evaluating the cause for hypertension, there was evidence of glomerulonephritis and multiple autoantibodies positivity including dsDNA. A diagnosis of lupus was made based on clinical and laboratory findings. Kidney biopsy revealed mesangial proliferative glomerulonephritis with predominant IgA deposits favouring IgA nephropathy. Additional features in the form of short stature with vertebral abnormalities and bilateral basal ganglia calcification led to evaluation of Type-1 interferonopathies. Sanger sequencing identified a novel compound heterozygous variants c.550C $>\mathrm{T}$ (p.Q184*) in exon 3 and c.740T>G (p.L247R) in exon 4 of $A C P 5$ gene. Parents were found to be carriers of the variants in ACP5 gene. Management included antihypertensive agents and symptomatic therapy. On follow-up, there was complete resolution of glomerulonephritis and normalization of blood pressure. This case report documents the classic phenotype comprising autoimmune, skeletal, and neurological abnormalities in spondyloenchondrodysplasia with immune dysregulation with a novel variant on Sanger sequencing in an Indian patient. This report also highlights the rare coexistence of IgA nephropathy in monogenic lupus.
\end{abstract}

Keywords Systemic lupus erythematous · Type-1 interferonopathies · Spondyloenchondrodysplasia with immune dysregulation · IgA nephropathy $\cdot$ Skeletal dysplasia $\cdot$ Basal ganglia calcification

\section{Introduction}

Monogenic lupus accounts for a small subset of systemic lupus erythematosus (SLE) and commonly caused by Type- 1 interferonopathies and defects in complement system [1]. Spondyloenchondrodysplasia with immune dysregulation (SPENCDI) is a Type-1 interferonopathy and a rare autosomal recessive skeletal dysplasia characterized by radiolucent

Shubha R. Phadke

shubharaophadke@gmail.com

1 Department of Medical Genetics, Sanjay Gandhi Postgraduate Institute of Medical Sciences, Lucknow, Uttar Pradesh, India

2 Department of Clinical Immunology \& Rheumatology, Sanjay Gandhi Postgraduate Institute of Medical Sciences, Lucknow, Uttar Pradesh, India

3 Department of Pathology, Sanjay Gandhi Postgraduate Institute of Medical Sciences, Lucknow, Uttar Pradesh, India vertebral and metaphyseal lesions. Central nervous system abnormalities include spasticity and cerebral calcifications and immunological abnormalities can range from autoimmune diseases like SLE, Sjogren syndrome to immunodeficiency. It is caused by biallelic mutation in ACP 5 gene which in turn results in deficient tartarate resistant acid phosphatase (TRAP). The authors report on a 15 -y-old female who initially presented with features suggestive of lupus and on work up was found to have neurological and skeletal features consistent with diagnosis of SPENCDI. A novel compound heterozygous variant was identified in ACP5 gene on Sanger sequencing.

\section{Report of Case}

Fifteen-year-old adolescent female from a nonconsanguineous family presented with recurrent bouts of fever for 2 mo, two episodes of seizures followed by progressive weakness of the left half 


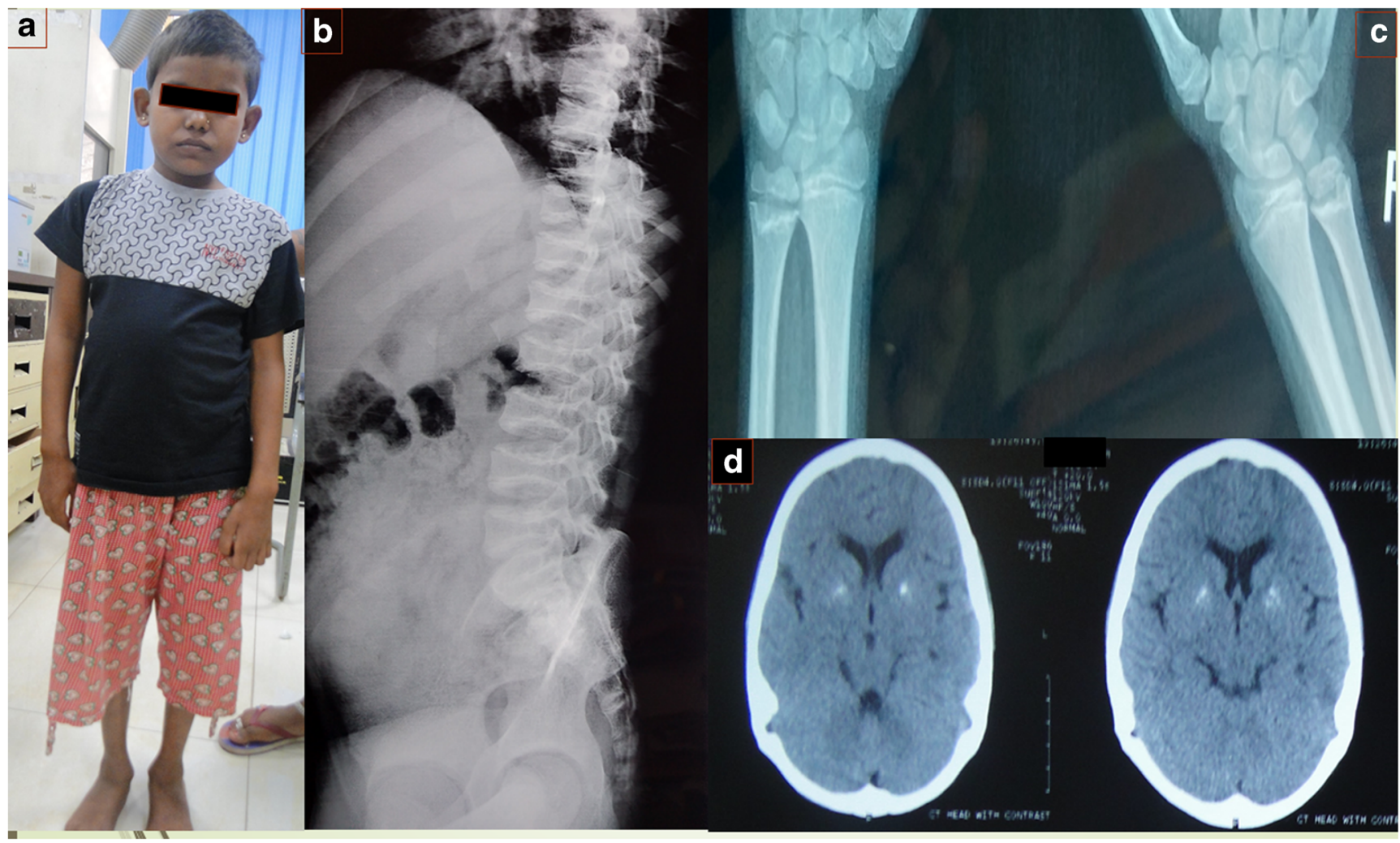

Fig. 1 a) 16-y-old proband, b) Radiograph of lateral view of spine showing irregularity involving the superior and inferior endplates of vertebrae, c) Radiograph wrist, showing mild irregularity involving distal

of body $10 \mathrm{~d}$ before presenting to the study facility centre (Fig. 1a). On evaluation, she had blood pressure of $210 / 160 \mathrm{mmHg}$ with no significant differences between the upper and lower limbs. Hemorrhagic stroke secondary to severe hypertension was suspected and a plain computed tomography (CT) was performed which revealed a large intraparenchymal hemorrhage involving right frontoparietal region. Fundus examination was suggestive of grade 4 hypertensive retinopathy with ischemic optic neuropathy. Hypertensive emergency was managed with intravenous labetalol.

Detailed history revealed a normal antenatal and perinatal period with normal developmental milestones. However, her scholastic performance was average. She had a past history of fever triggered generalised seizures at $9 \mathrm{y}$ of age for which no work up was done. She also had intermittent multiple painful large joint swellings for the past $2 \mathrm{y}$ which was not restricting her routine activities and usually resolves with antiinflammatory drugs. She had another episode of fever triggered generalized seizure $10 \mathrm{~d}$ before the cerebrovascular event. There was no history of recurrent infections, skin lesions, photosensitivity, or chronic systemic illnesses. She has not attained menarche till date. Family history revealed that the first female sibling expired at 6 mo of age due to febrile illness, one intrauterine death at $9 \mathrm{mo}$, the second sibling is a 12-y-old healthy male child with normal intelligence. metaphyseal ends of radius and ulna, d) Axial noncontrast CT showing bilateral basal ganglia calcification

On physical examination, her weight was $22 \mathrm{~kg}(-4.34 \mathrm{SD})$, height was $124 \mathrm{~cm}(-5.89 \mathrm{SD})$ with midparental height (MPH) of $145.5 \mathrm{~cm}$ and upper-segment to lower-segment ratio was 0.9 . Sparse hair was present. Sexual maturity rating corresponded to Tanner stage 2. Central nervous system examination revealed bilateral 6th and left upper motor neuron 7th cranial nerve palsy, left hemiparesis with visual acuity limited to projection of light in both eyes. Hepatosplenomegaly was evident (Liver: $6 \mathrm{~cm}$ below costal margin at midclavicular line with a span $9 \mathrm{~cm}$; left lobe: not palpable; spleen; tip palpable). There were no joint or skin abnormalities. Her baseline laboratory data is summarized in Table 1.

On evaluation for hypertension, renal doppler did not show any evidence of renal artery stenosis. Urinary metanephrine level was normal. Plasma renin activity and aldosterone levels were normal. 2D echocardiography was normal. Urine microscopy showed moderate proteinuria and hematuria. Renal biopsy revealed mesangioproliferative glomerulonephritis with segmental sclerosis and immunofluorescence showed granular mesangial deposits of IgA (3-4+), IgM (1-2+), kappa (1-2+) and lambda (3+) and absence of $\operatorname{IgG}, \mathrm{C} 3$ and $\mathrm{Clq}$ deposits with no immune deposits in the peritubular region. Electron microscopy showed presence of electron dense deposits in mesangium with absence of subendothelial deposits. These findings were more in favour of IgA nephropathy than lupus nephritis (Fig. 2a-d). Ultrasound 
Table 1 Baseline investigations of proband

\begin{tabular}{|c|c|}
\hline Investigations & Results \\
\hline Hemoglobin (g/dL) & 8.7 \\
\hline Total leucocyte count $\left(1000 / \mathrm{mm}^{3}\right)$ & $25.5(4-12)$ \\
\hline Differential count $(\mathrm{P} / \mathrm{L} / \mathrm{M} / \mathrm{E})(\%)$ & $51 / 40 / 6 / 3$ \\
\hline Total platelet count $\left(1000 / \mathrm{mm}^{3}\right)$ & $234(150-450)$ \\
\hline $\operatorname{ESR}(\mathrm{mm} / \mathrm{h})$ & $72(0-20)$ \\
\hline C-reactive protein $(\mathrm{mg} / \mathrm{dL})$ & $0.6(0-0.6)$ \\
\hline Uric acid (mg/dL) & $5.4(2.5-7)$ \\
\hline LDH (U/L) & $546(85-450)$ \\
\hline \multicolumn{2}{|l|}{ Kidney function test } \\
\hline Urea (mg/dL) & 45 \\
\hline Creatinine (mg/dL) & 0.4 \\
\hline \multicolumn{2}{|l|}{ Electrolytes } \\
\hline Sodium $(\mathrm{mEq} / \mathrm{L})$ & 134 \\
\hline Potassium (mEq/L) & 3.2 \\
\hline Calcium (mg/dL) & $8.9(9-11)$ \\
\hline Phosphorus (mg/dL) & $5.2(2.3-4.7)$ \\
\hline Magnesium (mg/dL) & $1.7(1.6-2.6)$ \\
\hline \multicolumn{2}{|l|}{ Liver function tests } \\
\hline Total bilirubin (mg/dL) & 0.7 \\
\hline SGOT/SGPT (U/L) & $60 / 71$ \\
\hline Serum albumin $(\mathrm{g} / \mathrm{dL})$ & 3.9 \\
\hline PT/aPTT & Normal \\
\hline \multicolumn{2}{|l|}{ Urine analysis } \\
\hline Protein & $3+$ \\
\hline RBC (cells) & $6-8$ \\
\hline WBC (cells) & $2-4$ \\
\hline $24 \mathrm{~h}$ urine protein $(\mathrm{g} / 1100 \mathrm{~mL})$ & 1.1 \\
\hline $24 \mathrm{~h}$ urine creatinine $(\mathrm{g} / 1100 \mathrm{~mL})$ & 0.132 \\
\hline \multicolumn{2}{|l|}{ Immunological } \\
\hline ANA & $\begin{array}{l}\text { 4+ cytoplasmic ANA on Hep2 } \\
\text { cells } 1: 100\end{array}$ \\
\hline dsDNA (IU/mL) & $67.5(0-30)$ \\
\hline ENA & $\mathrm{SS}-\mathrm{A} / \mathrm{R} 052 \mathrm{kD}+$ \\
\hline APLA & Negative \\
\hline Lupus anticoagulant & Negative \\
\hline $\mathrm{C} 3(\mathrm{mg} / \mathrm{dL})$ & $105(60-120)$ \\
\hline $\mathrm{C} 4$ (mg/dL) & $29.6(15-25)$ \\
\hline IgM (mg/dL) & $60.9(60-280)$ \\
\hline $\operatorname{IgA}(\mathrm{mg} / \mathrm{dL})$ & $621(90-450)$ \\
\hline $\operatorname{IgG}(\mathrm{mg} / \mathrm{dL})$ & $3590(800-1800)$ \\
\hline \multicolumn{2}{|l|}{ Endocrine } \\
\hline TSH (mIU/L) & $20.3(0.5-5.5)$ \\
\hline iPTH & 24.3 \\
\hline FSH (IU/L) & 1.17 \\
\hline Aldosterone (nmol/L) & $0.5(0.1-0.5)$ \\
\hline PRA (ng/mL/h) & $15.3(0.8-1)$ \\
\hline $\begin{array}{l}\text { Urine metanephrine and } \\
\text { normetanephrine }\end{array}$ & Normal \\
\hline
\end{tabular}

Table 1 (continued)

\begin{tabular}{ll}
\hline Investigations & Results \\
\hline Culture and sensitivity & \\
Urine & Sterile \\
Blood & Sterile \\
\hline
\end{tabular}

ANA Antinuclear antibodies; APLA Antiphospholipid antibodies; aPTT Activated partial thromboplastin time; $C$ Complement; $d s D N A$ Doublestranded DNA; ENA Extractable nuclear antigen antibodies; ESR Erythrocyte sedimentation rate; FSH Follicle stimulating hormone; $I g$ Immunoglobulin; iPTH intact-parathyroid hormone; $L D H$ Lactate dehydrogenase; $P R A$ Plasma renin activity; $P T$ Prothrombin time; $R B C$ Red blood corpuscles/cells; SGOT Serum glutamic-oxaloacetic transaminase; $S G P T$ Serum glutamic-pyruvic transaminase; TSH Thyroid stimulating hormone; $W B C$ White blood corpuscles/cells

of the abdomen showed hepatomegaly and medical renal disease with loss of corticomedullary differentiation. CT angiogram of abdomen detected bilateral normal-sized kidney with striated nephrogram on cortical phase (probably secondary to vasculitis), cholelithiasis, hepatosplenomegaly with osteopenia of visualized bones and $\mathrm{H}$-shaped configuration of visualised vertebral bodies. She had positive antinuclear antibodies (4+) and double stranded DNA [67 IU/mL; $(0-30)]$ with normal C3 and C4 levels. She fulfilled the Systemic Lupus International Collaborating Clinics (SLICC) criteria for SLE (5/11). On review of previous $\mathrm{CT}$ film, an incidental bilateral basal ganglia calcification was noted (Fig. 1d).

In view of severe stunting with vertebral abnormalities and bilateral basal ganglia calcification, Type-1 interferonopathies associated with monogenic lupus were considered. Endocrine evaluation showed hyperthyrotropinemia [TSH: $20.3 \mathrm{mIU} / \mathrm{L}$, fT4: $0.7 \mathrm{ng} / \mathrm{dL}(0.8-1.8)]$ and low follicle stimulating hormone (FSH: 1.17 IU/L). Karyotype was 46, XX. Skeletal survey showed irregularity involving the superior and inferior endplates of thoracic and lumbar vertebrae and distal metaphyseal ends of radius and ulna (Fig. $1 \mathrm{~b}$ and c).

Sanger sequencing was performed after getting informed consent from parents. The patient was found to be compound heterozygous for a nonsense variant $[\mathrm{c} .550 \mathrm{C}>\mathrm{T}(\mathrm{p} . \mathrm{G} \ln 184 *)]$ in exon 3 and a missense variant [c.740T>G (p.Leu247Arg)] in exon 4 of ACP5 gene (Fig. 2e and f). Both the variants were absent in population databases (gnomAD, 1000 Genome) and disease database (Clinvar, HGMD). Both the variants were predicted to be deleterious by prediction tools (MutationTaster, SIFT, Provean and Polyphen). Segregation analysis revealed both the parents to be carriers of the variants identified in ACP5 gene.

The patient subsequently required three antihypertensives; amlodipine, enalapril and labetalol. With renal biopsy favouring IgA nephropathy, daily monitoring of kidney function and proteinuria was done for progression of the disease. Since blood pressure got controlled, kidney function remained 


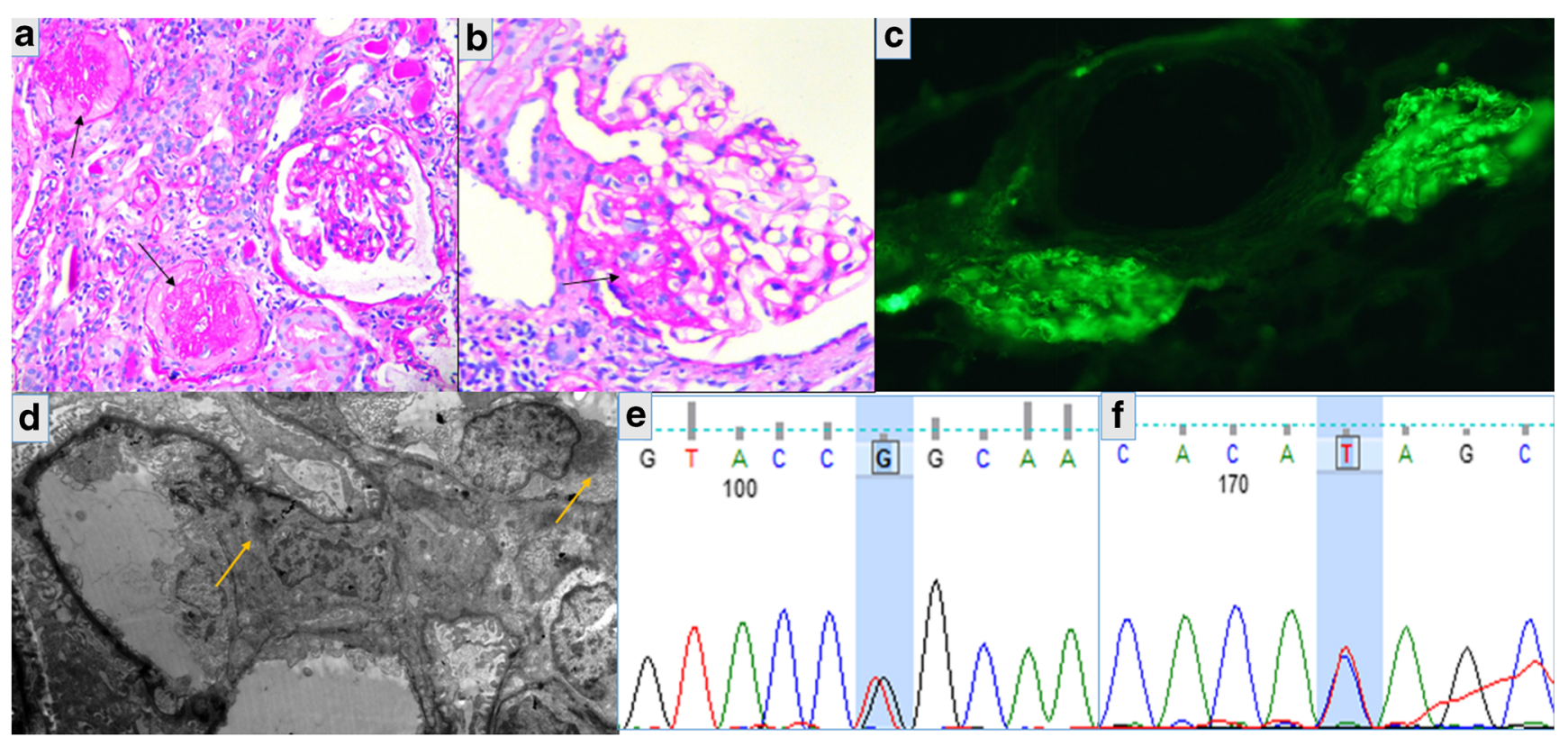

Fig. 2 a) Light microscopy (200x original magnification) showing 3 glomeruli with global sclerosis in two of them (Thin arrow), b) A single glomerulus with mild segmental mesangial proliferation (Thin arrow), c) Immunofluorescence staining for $\operatorname{IgA}$ (400x original magnification): $3-4+$ granular deposits of IgA in the mesangium, d)
Transmission electron microscopy showing few electron dense mesangial deposits (yellow arrow), e) Sanger sequencing of proband showing missense variant [c.740T $>\mathrm{G}$ (p.Leu247Arg)] in exon 4 and f) nonsense variant $[\mathrm{c} .550 \mathrm{C}>\mathrm{T}$ (p.Gln184*)] in exon 3 of $A C P 5$ gene stable and proteinuria settled with symptomatic management, immunosuppressive therapy was not instituted. At discharge, she had residual deficits on left half of body for which physiotherapy was advised. On follow-up visit, she had spastic hemiparesis and her vision improved to counting of fingers. Repeat urine microscopy showed no evidence of hematuria and proteinuria. The antihypertensive drugs was tapered to a single agent. In view of the patient fulfilling SLICC criteria, hydroxychloroquine was added to prevent disease flare up. On follow-up, there was history of recurrent bouts of fever which on investigation was not suggestive of any infectious etiology and fever was managed with antipyretics.

\section{Discussion}

SLE is usually considered as a multifactorial disorder and monogenic lupus accounts for a small subset of lupus patients. However, identification of monogenic forms has important prognostic and treatment implications. Early onset disease in prepubertal period, evidence of mendelian inheritance or strong family history, less typical manifestations such as severe skin, neurologic or joint disease and refractoriness to standard therapy should raise the suspicion for monogenic lupus. Majority are caused by defect in genes of complement system and Type-1 interferon regulation [1]. Type-1 interferonopathies include Aicardi-Goutières syndrome (AGS) and SPENCDI [2]. These disorders share few phenotypic similarities, like neurological impairment including brain calcifications, multisystem inflammation, and autoimmunity. However, neurological impairment is severe in AGS and presence of skeletal dysplasia can aid in differentiation of SPENCDI from AGS.

SPENCDI is caused by biallelic mutation in ACP5 gene which in turn results in deficient tartarate resistant acid phosphatase (TRAP). TRAP is a protein that is abundantly expressed in osteoclasts, macrophages, and dendritic cells [3] and is a primary substrate of osteopontin. Osteopontin in turn regulates the production of Type-1 interferon (IFN) by dendritic cells [4]. Deficient TRAP activity results in hyperphosphorylation of osteopontin. This is postulated to cause increased bone resorption resulting in skeletal abnormalities and immune dysregulation resulting from increased Type-1 IFN production.

In SPENCDI, autoimmune manifestations are the most common presentation [5]. In the largest case series by Briggs et al., autoimmune manifestations were present in $85 \%$ of study population with many individuals exhibiting more than three manifestations. Autoimmune thrombocytopenic purpura (AITP) (46\%) and SLE were the commonest autoimmune manifestations and seven individuals exhibited both the condition. In patients with SLE, only $36 \%$ fulfilled the ACR diagnostic criteria. Recurrent bouts of fever of unknown origin, vasculitis, Raynaud phenomenon and Sjögren syndrome were other autoimmune phenomenon observed in their study. These individuals were also found to be at risk for multiple autoimmune complications (one individual developed eight autoimmune diseases over a period of $27 \mathrm{y}$ ). On 
the contrary, individuals with autoantibody positivity without clinical evidence of disease were also observed. These findings emphasize the importance of stringent lifelong monitoring of these patients.

Neurological manifestations generally evolve over time in SPENCDI. While the authors did not observe any developmental delay or pre-existing spasticity in their patient, mild intellectual disability and seizures with basal ganglia calcification was evident. The skeletal manifestations can range from platyspondyly with irregular superior and inferior endplates and extensive metaphseal and diaphyseal involvement of long bones to minimal metaphyseal involvement of long bones thus explaining the pleiotropic nature of this disorder.

Though the patient had clinical and laboratory features of lupus, the immunofluorescence was favouring IgA nephropathy due to presence of mesangial hypercellularity and dominant deposits of $\operatorname{IgA}$ in the glomerulus along with IgM, kappa and lambda deposits. Lupus nephritis, on the other hand, exhibits glomerular, vascular (wire loop lesions), and tubulointerstitial lesions with deposition of polyclonal immunoglobulins, mainly IgG and fractions of complement $\mathrm{C} 1 \mathrm{q}, \mathrm{C} 3$ and $\mathrm{C} 4$ [6]. Though mesangial $\operatorname{IgA}$ deposition can be found in the subtype of lupus nephritis, absence of $\mathrm{C} 3, \mathrm{C} 1 \mathrm{q}, \mathrm{IgG}$ goes more in favor of IgA nephropathy. Recent studies have evaluated the role of a monoclonal antibody KM55 in specifically recognizing galactose-deficient IgA1 in serum and glomeruli. Utilization of such assays when available can end such diagnostic odyssey [7]. The presence of IgA nephropathy in patients with SLE has been previously reported in less than 10 cases worldwide $[8,9]$. The coexistence of IgA nephropathy and SLE in the authors' patient can be explained by generalized dysfunction of immune system and propensity to develop multiple autoimmune manifestations in this disorder. Identification of this atypical finding also has important prognostic and treatment implications as immunosuppressive agents are often required in lupus nephritis, unlike IgA nephropathy, where the management is usually conservative.

Identification of monogenic causes for SLE is necessary as it has important treatment implications. For monogenic SLE, conventional treatments with steroid or disease modifying antirheumatic drugs can be tried. Recently, several clinical trials using JAK $1 / 2$ inhibitors showed promising results for monogenic autoimmune diseases [10].

To conclude, this case-report highlights the characteristic findings of SPENCDI in the form of skeletal, neurological, and autoimmune manifestations and a novel compound heterozygous variant in the $A C P 5$ gene in an Indian patient. This case-report also highlights a rare coexistence of IgA nephropathy in a patient with monogenic lupus. Identification of monogenic susceptibility to lupus has important clinical implications in terms of screening for other subclinical comorbid autoimmune disorders and for accurate genetic counseling.

Acknowledgements The authors would like to thank the parents and Mrs. Suzena (PhD scholar) for her support in primer designing and Mr. Shashank Shende (technical officer) for helping us in performing Sanger sequencing.

Authors' Contributions HS and SRP conceived the idea of the project, $\mathrm{AA}$ and HG was involved in the clinical case management. AG and MJ played an important role in evaluating the pathological aspect of this case. HS and SRP was involved in the molecular analysis and interpretation. SRP, AA and MJ finally critically reviewed the manuscript and SRP will act as guarantor for the paper.

The manuscript has been read and approved by all the authors and the requirements for authorship as stated earlier in this document have been met, and that each author believes that the manuscript represents honest work.

\section{Compliance with Ethical Standards}

Conflict of Interest None.

\section{References}

1. Omarjee O, Picard C, Frachette C, et al. Monogenic lupus: dissecting heterogeneity. Autoimmun Rev. 2019;18:102361.

2. Weber GF, Zawaideh S, Hikita S, Kumar VA, Cantor H, Ashkar S. Phosphorylation-dependent interaction of osteopontin with its receptors regulates macrophage migration and activation. J Leukoc Biol. 2002;72:752-61.

3. Shinohara ML, Lu L, Bu J, et al. Osteopontin expression is essential for interferon-alpha production by plasmacytoid dendritic cells. Nat Immunol. 2006;7:498-506.

4. Alperin JM, Ortiz-Fernández L, Sawalha AH. Monogenic lupus: a developing paradigm of disease. Front Immunol. 2018;9:2496.

5. Briggs TA, Rice GI, Adib N, et al. Spondyloenchondrodysplasia due to mutations in ACP5: a comprehensive survey. J Clin Immunol. 2016;36:220-34.

6. da Silva LS, Almeida BL, de Melo AK, de Brito DC, Braz AS, Freire EA. IgA nephropathy in systemic lupus erythematosus patients: case report and literature review. Rev Bras Reumatol Engl Ed. 2016;56:270-3.

7. Zhang K, Li Q, Zhang Y, et al. Clinical significance of galactosedeficient IgA1 by KM55 in patients with IgA nephropathy. Kidney Blood Press Res. 2019;44:1196-206.

8. Horino T, Takao T, Terada Y. IgA nephropathy in a patient with systemic lupus erythematosus. Lupus. 2010;19:650-4.

9. Corrado A, Quarta L, Di Palma AM, Gesualdo L, Cantatore FP. IgA nephropathy in systemic lupus erythematosus. Clin Exp Rheumatol. 2007;25:467-9.

10. Kim H, Brooks KM, Tang CC, et al. Pharmacokinetics, pharmacodynamics, and proposed dosing of the oral JAK1 and JAK2 inhibitor baricitinib in pediatric and young adult CANDLE and SAVI patients. Clin Pharmacol Ther. 2018;104:364-73.

Publisher's Note Springer Nature remains neutral with regard to jurisdictional claims in published maps and institutional affiliations. 